Uluslararası Mühendislik

Cilt/Volume:11 Sayı/Issue:3 Aralık/December 2019

Araştırma Makalesi / Research Article

\title{
Machine Learning of Kidney Tumors and Diagnosis and Classification by Deep Learning Methods
}

\author{
Fuat Türk1 $^{1}$ (D), Murat Lüy² ${ }^{\text {iD }}$, Necaattin Barış̧ı ${ }^{3}$ \\ ${ }^{1}$ Bilgisayar Müh./Fen Bilimleri Ens. Kırıkkale Üniversitesi, 71450 Kırıkkale, TÜRKIYE \\ ${ }^{2}$ Elektrik - Elektronik Müh. Kirıkkale Üniversitesi, 71450 Kırıkkale, TÜRKIYE \\ ${ }^{3}$ Bilgisayar Müh. Gazi Üniversitesi, Ankara, TÜRKIYY
}

\begin{abstract}
Kidney cancer is difficult to diagnose and it can be quite complicated for physicians to diagnose. In this study, while providing information about multiple sources to help people who are dealing with the challenges of the diagnosis of kidney cancer, in order to serve as a guide the principles of kidney cancer are tried to be explained. In recent years, many new methods of treatment have been developed for kidney cancer, and some are under development by scientists. These studies provide treatment information that offers new hope to the lives of kidney cancer patients. In this study, it is aimed to get acquainted with kidney cancer cells by using machine learning, and deep learning algorithms. In this way, an application can be developed to guide patients and physicians through early diagnosis and classification.
\end{abstract}

Key Words

Deep learning, Machine learning, Kidney cancer 


\section{Introduction}

Kidney cancer is expressed in medical language as renal cell carcinoma. The word 'renal' comes from the Latin word renalis.. kidney cancer can occur in various ways. (American Cancer Society 2008).

While the diagnosis of some patients occurs after cancer has spread to the body, in some patients it can give symptoms without spreading to the body. If the patient is talking about metastatic spread, surgical or systematic medical treatment may result depending on the condition. If only surgery is desired, additional treatments may be recommended to treat metastatic spread or to postpone the progression of cancer. As with many cancers, kidney cancers usually occur by chance. however, some external factors such as smoking and obesity also increase the likelihood of kidney cancer. During treatment of patients, it is important to know which factors increase the risk of kidney cancer, but seeking past behavior does not benefit healing. The smoking habit of the patient does not necessarily indicate the risk of cancer. Genetic factors may be associated with an increased risk of developing kidney cancer. For example, a genetic disorder called von Hippel-Lindau (VHL) may increase the risk of developing kidney cancer. (American Cancer Society, 2008).

\section{Kidney}

In a normal person, the kidneys are located on the right and left of the body, towards the back of the rib cage. The kidneys are covered with protective adipose tissue. There is an adrenal gland at the top of each kidney. In a normal person, the kidneys may not have two, but it may be possible to live with a single kidney. In the kidneys, there is a membrane called a capsule. This membrane is flexible, and when a kidney tumor is seen, the membrane is stretched. If the tumor is diagnosed early, it may remain in the capsule and treatment may be possible by surgical removal of the kidney.

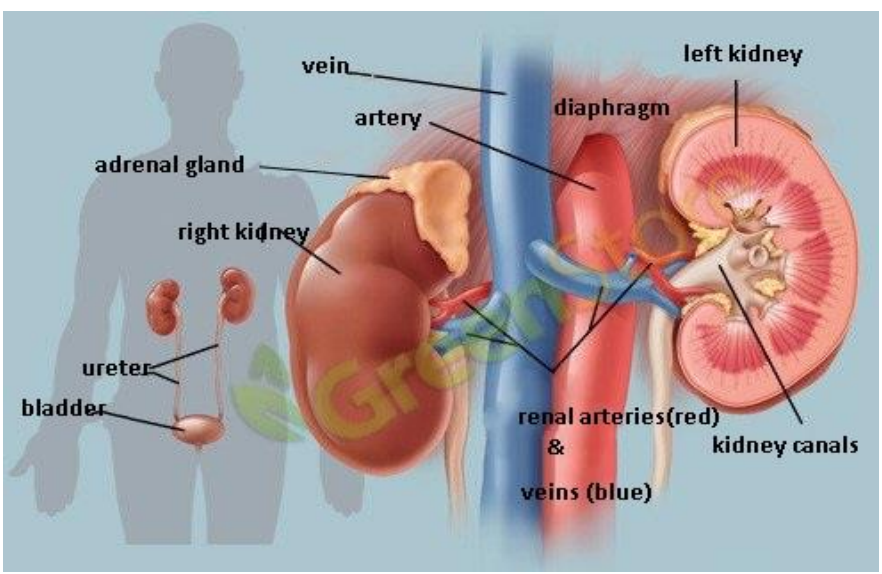

Figure 1.The body Structure of the Kidneys

\subsection{Overview of Kidney Cancer}

According to the American Cancer Society (ACS), more than one million new cancer cases are seen in the United States each year. especially recently, the proportion of kidney cancer cases accounts for almost $4 \%$ of the total cancer cases. In 2008 , ACS reported more than 54,000 people diagnosed with kidney cancer (Uroweb, 2018).

In recent years, the incidence of kidney cancer in our country and in the world is increasing rapidly. Therefore, kidney cancer surgery operations also increase. In 2012, 84,400 new cases of kidney cancer have been reported in European Union countries (Muratbinbay, 2018). According to Ministry of Health data for 2013, cases of kidney tumors in Turkey the number of which was 6534 accounted for 3 percent of all cancer patients (Onkoloji, 2018).

Kidney cancer is twice as common in men as in women, but this difference is gradually decreasing. In 2008, approximately 13,000 people lost their lives due to kidney cancer. However, it is known that between 100,000 and 200,000 people in the United States survive kidney cancer and survive. These statistics were calculated for all types of kidney cancer including both adults and children.

In recent years, the incidence of kidney cancer in our country and in the world is increasing rapidly. Therefore, kidney cancer surgery operations are also increasing.

\subsection{Kidney Cancer Diagnosis}

Often, most kidney tumors are found incidentally during X-ray or ultrasound imaging for non-tumor reasons (when they go to the hospital for another complaint). There are several risk factors that may lead to kidney cancer. These factors may include smoking, obesity, cadmium, and petroleum by-products (eg petroleum), which nearly double the person's cancer risk. People with kidney cancer in the family have a higher risk of developing this cancer than normal people. The most common symptom of kidney cancer is painless bleeding in urine known as hematuria. This symptom occurs in about $20 \%$ to $25 \%$ of patients. other signs of fat in kidney cancer patients may be abdominal distension, hard swelling or thickening, which can be seen or felt when the tumor grows under the skin. There may be pain or pressure on the back or side. Kidney cancer is normally seen between the 
ages of 40 to 60 years. Because back pain is common among people over the age of 40, this pain is often overlooked and therefore the presence of kidney cancer is often unaccounted for (Onkoloji, 2018).

If the tumor has spread to other organs, the symptoms may vary according to the affected organ, and patients may notice symptoms of unknown cause such as weight loss, fever, anemia or high blood pressure.

Even though cancer begins in the kidney and spreads to other organs, it is still considered to be kidney cancer. The main diagnostic methods are as follows.

- Computed Tomography (CT)

- Magnetic Resonance Imaging (MRI)

- Bone Screening

-Positron Emission Tomography (PET) Screening

- Ultrasonography (Ultrasound or US)

- Biopsy

In addition;

- Urine test

- Blood tests

\subsection{Renal Cancer Classification}

\section{Tumor:}

T-1: It is divided into two sub-groups

T1a: Tumor in the kidney (not exceeding the kidney capsule) smaller than $4 \mathrm{~cm}$.

T1b: Kidney limited (not exceeding the kidney capsule) size between 4-7 cm.

\section{T-2:}

T2a: Tumor in the kidney (not exceeding the kidney capsule), greater than $7 \mathrm{~cm}$, but less than $10 \mathrm{~cm}$ in diameter

T2b: Tumor diameter greater than $10 \mathrm{~cm}$

T-3: It is divided into three sub-groups.

T3a: Tumor that is over the kidney capsule and infiltrated the adrenal gland

T3b: Tumor that is over the kidney capsule and infiltrated the kidney vein

T3c: Tumor tissue exceeding the kidney capsule and vena cava.

T-4: It has exceeded the velamen (Gerota fascia). Which surrounds the adipose tissue around the kidney.

\section{Lymph Gland:}

Nx: Regional lymph glands have not been evaluated.

N0: Tumor spread in regional lymph glands that is; metastasis does not exist.

$\mathrm{N} 1$ : Tumor spread in regional lymph glands that is; metastasis exists.

\section{Remote Metastasis:}

M0: The Spread of cancer to distant organs does not exist.

M1. The Spread of cancer to distant organs exists (Tibeter, 2018).

The classifications above are shown in Table 1.

Table 1. Classification of Kidney Tumors.

\begin{tabular}{cccc}
\hline Phase & T & N & M \\
\hline I & T1 & N0 & M0 \\
II & T2 & N0 & M0 \\
III & T1 or T2 & N1 & M0 \\
& T3 & N0 or N1 & M0 \\
IV & T4 & Any N & M0 \\
& Any T & Any N & M1
\end{tabular}




\section{Artificial Intelligence Learning Methods}

Artificial intelligence; It is a set of software and hardware systems with many capabilities such as exhibiting behaviors like human, digital reasoning, motion, speaking and sound detection. In other words, artificial intelligence; provides computers to think like people. It would be wrong to consider artificial intelligence in a single title. Concepts such as machine learning, deep learning (as seen in Figure 2) are the inclusive terms that constitute artificial intelligence. It would be more useful to analyze the chronological order shown in Figure 2. to understand the difference between artificial intelligence, machine learning, and deep learning terms.

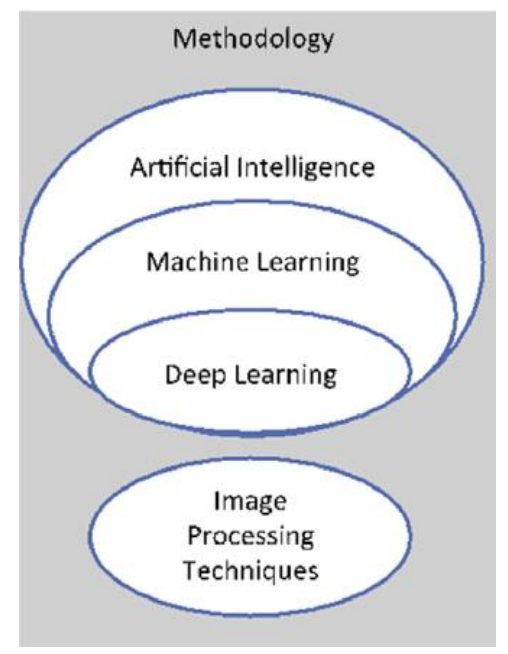

Figure 2. Deep learning methodology

\subsection{What is Machine Learning?}

Machine learning is expressed as the name of computer algorithms that model a problem based on available data. The model, which was created with the existing data set and algorithm, was designed to get the highest performance (Mehmet Akif Ersoy Ü., 2017).

The types of algorithms in Machine Learning are generally considered in two main categories. these are referred to as Supervised Learning and Unsupervised Learning.

\section{Supervised Learning}

Controlled learning or controlled learning is to learn the function of obtaining the desired output set $\mathrm{Y}$ from the $\mathrm{X}$ input data set. Supervised learning includes a tagged training data. Training data includes each sample input and available outputs. There is also a connection between input and output. We are aware of how the correct output should be in the data set in supervised learning. In supervised learning, the collected observations about the concept to be learned are given to the learner as an educational setting. They are also given the desired output values that are asked for each sample training set. Using this information, a relationship is established between input and output. By using the obtained correlation model, the Y outputs corresponding to the future X observation can be estimated (as shown in Figure 3).

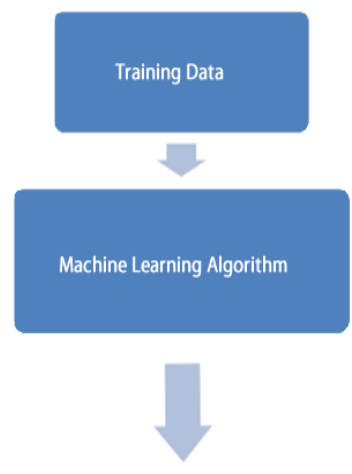

\section{New Data}
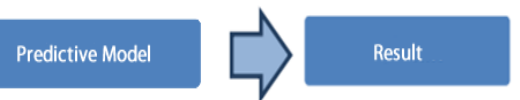

Figure 3. Stages of Machine Learning Observed 


\section{Unsupervised Learning}

In the case of unsupervised learning, only the $\mathrm{X}$ input set is applied to the system. Although there is no $\mathrm{Y}$-set associated with $\mathrm{X}$, it is tried to be predicted on the system. Unsupervised learning allows researchers to gain insight into the nature of unknown problems. (Eskisehir, 2017).

Other major types of algorithms used in Machine Learning are:

- Bayesian

- Decision trees

- Dimensionality reduction

- Clustering

- Deep learning

- Community (ensemble)

- Neural networks

- Regularization

- Rule- based system

- Regression

\subsection{What is Deep Learning?}

Deep learning is a kind of machine learning of the human brain by working on big data, starting with a specific rule and discovering the patterns in that big picture, creating new patterns.

It automates the algorithm creation process. In the examination of the data, it is not planned before where to look or what to conclude. It uses neural networks, statistics, operation research and physics methods to make sense of these data by accessing confidential information in the data.

Fig. 4, Fig.5 shows some images related to deep learning for a better understanding of the subject (Eskisehir, 2017).

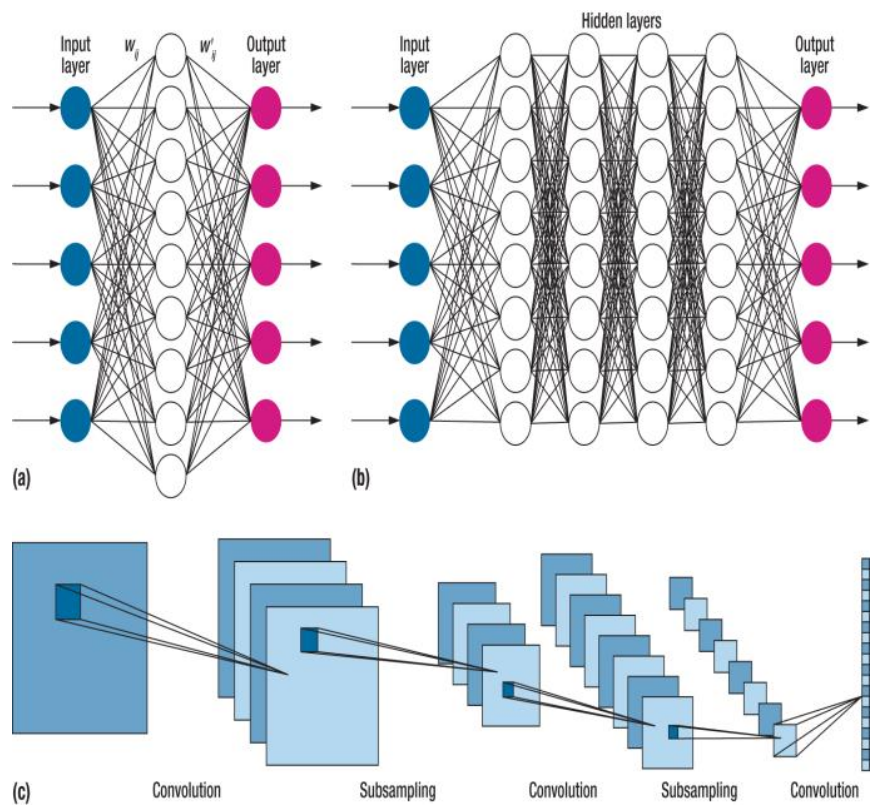

Figure 4. Deep learning networks

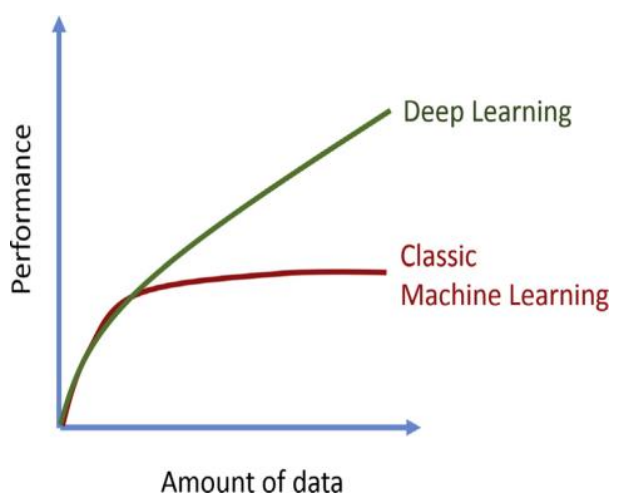

Figure 5. Training with deep learning 


\subsection{Deep Learning Architectures}

\subsubsection{Convulsive Neural Networks}

Convolutional Neural Networks (Convolution Neural Network-CNN) is a type of multi-layer sensors (Multi-Layer PerceptronMLP). Cells in the center of vision are separated into sub-regions in a way to involve the whole image, it is thought that simple cells concentrate on edge-like properties, but the complex cells concentrate on the whole image with larger receptors. The CNN algorithm, which is a forward neural network, was put forward inspired by the vision center of the animals. The mathematical convolution process here can be thought as a response from a neuron to its stimulus. (Fukushim, 1980). CNN algorithms are applied in many different fields such as natural language processing (NLP), biomedical, especially in the field of image and sound processing (Hubel and Wiesel, 1968, Lecun et al., 1998).

\subsubsection{Recurrent Neural Networks}

First of all, the Simple Recurrent Network (SRN) was designed by Jeff Elman. For each word used in the sentence structure simulation of Elman, the name and verb categories are separated cleanly on the average pattern clustering over the hidden patterns. In addition to this, clusters such as live-inanimate distinctions between names, even human-animal, hunter-predator between animals are divided (Elman, J. L., 1991). Recurrent Neural Network (RNN) is a type of artificial neural network in which the connections between the units form a directed loop. This loop creates a network state that allows it to display dynamic temporal behavior. In contrast to feedforward neural networks, RNNs can use input memory to process random sequences of inputs (Mikolov, 2010).

\subsubsection{Restricted Boltzmann Machines}

Restricted Boltzmann Machines (RBM) was first described by Harmonium in 1986 (Smolensky, 1986), but twenty years later (2006) it was expressed as a fast learning algorithm by Geoffrey Hinton et al.. RBM is a productive random neural network that can learn probability distribution on the input set. RBM is a type of Boltzmann Machines. They consist of two-part graphs that are visible and hidden with the symmetrical connection between them. There is no connection between the nodes within a graph (Hinton and Salakhutdinov, 2006). In contrast, Unrestricted Boltzmann Machines are also connected between hidden units. This restriction allows for more effective training algorithms than those available for the general class of Boltzmann machines (Hinton and Salakhutdinov, 2009).

\subsubsection{Deep Faith Networks}

Deep Belief Networks (DBN), introduced by Geoffrey Hinton, are defined as the stack of RBMs given in the previous section. Each RBM layer is bound to both the previous and next layers. However, any layer has no horizontal communication with the nodes. It is capable of clustering for grading or uncontrolled learning with a softmax layer as being the last layer ( Carreira, and Hinton, 2005). DBN architectures have been applied to image recognition and production (Hinton and Osindero, 2006, Ranzato and Huang, 2007).

\subsubsection{Deep Auto-Encoders}

Auto-encoders (AE) called diabolo network is a special artificial neural network used for unsupervised learning. The AE aims at learning a representation (coding) for a size reduction for a data set. $\mathrm{AE}$ is a feed-forward neural network that aims to learn the best features from the compressed representation of the input data. In the AE logic, weights are changed until seeing the same input as the output after the input data encryption-decryption process. When the goal is reached, input data is represented by the number of nodes in the hidden layer. Deep or stacked auto-coders (Deep / Stacked Auto encoder-DAE) are neural networks of multiple layers of AEs, in which the outputs in each layer are connected to the inputs of the successive layer (Bengio et al., 2006, Krizhevsky and Hinton, 2011).

The input sequence is encoded in the first hidden layer and the "feature I" sequence is obtained. The sequence of properties obtained from the next hidden layer is given as input and as a result of the encryption "feature II" sequence is obtained. The feature II sequence is given as input to the softmax classifier in the final layer as the feature vector, the classification process is performed with this vector (Seker and Gurkan, 2017, Seker et al. 2017).

\section{Related Works}

\subsection{Works About Machine Learning}

In nephrology, the Prospective Prenatal Hydronephrosis Database was created and disease diagnose has been done by using the Microsoft $₫$ Azure Machine Learning Studio for data sharing in the Estimated Analytical and Modeling Machine Learning Technology study. As a result of the study, the optimized model has reached an accuracy of 0.87 and 0.80 and it has become applicable in a web-based environment (Lorenzo et al., 2018).

In "textural differences between renal cell carcinoma subtypes" study, three main subtypes of renal cell carcinomas (RCC) were classified as externally approved, reproducible and generalizable models to differentiate them using machine learning methods. WEKA software was used for this classification. As a result of this study, it is concluded that; a machine learning based method can distinguish the qCT-TA, cc-RCC from the cc-RCC with a satisfactory performance by overcoming the other methods (Kocak et al., 2018).

In the early detection study of Ureter pelvic region obstruction (the portion that passes through the kidney from the pelvis to the urine tube), machine learning methods significantly increased the diagnostic accuracy of clinically significant hydronephrosis. 
By providing a method of machine learning for early detection of these serious cases, it has helped the clinical decision-making process and has the potential to reduce the number of diuresis renograms required for diagnosis (Emily et al., 2018).

In the study of the diagnosis of chronic kidney disease by using machine learning, prediction models using machine learning methods such as K-closest neighbors (KNN), support vector machine (SVM) and logistics are presented. From the experimental results, it was found that the SVM classifier gave the highest accuracy. In addition, SVM has the highest precision after training and testing with the recommended method. Therefore, it was concluded that the SVM classifier was suitable for predicting chronic kidney disease (Anusorn, 2016).

In a study named "Transfer Learning for Diagnosis of Congenital Abnormalities of the Kidney and Urinary Tract in Children Based on Ultrasound Imaging Data", the classification of ultrasound (US) kidney images for the diagnosis of kidneys and urinary tract in children has been shown to be a challenging task. In this study, transfer-based learning method was proposed to extract imaging characteristics from US kidney images to improve kidney congenital anomalies and urinary tract (CAKUT) diagnosis in children.

The support vector machine classifiers are built on different sets of traits, including transfer learning features, conventional imaging features, and their combination. Experimental results showed that the combination of transfer learning characteristics and traditional imaging properties yielded the best results. In this paper, a transfer-based learning method has been proposed for the diagnosis of CAKUT based on US kidney images (Qiang et al., 2018).

In the early estimation of chronic kidney disease using predictive analytical machine learning, the capabilities of various machine learning methods for early detection of Chronic Kidney Disease were investigated. This topic has been studied extensively, however, the relationship between data parameters and the target class attribute has been investigated and machine learning methods have yielded very successful results for early estimation (Ahmed, Aljaaf, 2018).

In this study, the machine learning algorithms were compared with other methods (such as ANN) in the study of chronic Different Classification Methods in the Detection of Chronic Kidney Disease and the Combination of Different Community Algorithms to Classification Performance Hast. In this study conducted on 400 patients, machine learning algorithms absorbed the prediction with the successful classification of $96.25 \%$ (Eroglu and Palabas, 2016).

Chronic kidney disease was determined by using machine learning algorithm in the study of renal Detection of Chronic Kidney Disease Using Group Classifiers “. As a result of the studies, the disease has been achieved by achieving 99\% success (Dogruyol and Aydin, 2017).

Machine learning in medicine: Machine learning algorithms are used in the study of Calculation of minimum dose of hemodialysis using neural networks. The fact that artificial intelligence methods have not been studied before has also made this study important. The results of the study were very promising in terms of medicine. This study, however, points to the efficiency of working with more data (Monika and Uvais, 2003).

A study was carried out with machine learning techniques for the classification and diagnosis of diabetes patients. He trained the model using six different classification algorithms, achieving a precision equal to 0.757 and the best feature removed to 0.762 after the selection step (Mercaldo, Nardone, Santone, 2017).

In addition to known methods, machine learning algorithms (support vector machine) have been used in the study called algorithm Diagnosis of chronic kidney disease based on ultrasonography “. $82 \%$ success was achieved in the study. In order to determine the stage of chronic kidney disease (CKD) in potential or at risk, it is emphasized that blood test procedures are important and also a cheap, fast and high sensitivity detection system has been developed. This system may make it easier for physicians to diagnose early CKD patients to provide appropriate clinical treatment. However, it is stated that the samples collected in this study are not sufficient yet. In the near future, increasing training samples and applying the correct test methods can improve our system performance positively and a better and more reliable estimation system is expected (Chi-Jim et al. , 2014 ).

Machine learning algorithms were used for the study of kidney detection and the segment in a rapid way with 3D ultrasound images. In 3D ultrasound images, a method for dividing the kidneys into segments is presented. The main difficulties were high variability in renal appearance, the frequency of artifacts (shadows, speckle noise, etc.) and a strong restriction in the calculation. The algorithm was supported by a database of 480 3D images with a support vector machine (SVM) based detection algorithm and then a model-based deformation technique. Because severe pathologies induce strong deformations of the kidneys, the proposed method includes intuitive interaction functions that allow the user to improve the result in a few clicks. It was verified by 120 base and tests. Over 360; an excellent segmentation was achieved in 50\% of the cases, with less than 3 clicks in $90 \%$ of cases (Roberto, Remi, Ketan, Vincent, 2015).

"Integration of Multimodal RNA-Seq Data for Prediction of Kidney Cancer Survival", in this study, two different machine learning algorithms (KNN and SVM) were compared in terms of results. For the 4 parameters of the study (Gen, exon, isoform, coupling modalities), KNN cross-validation provided accuracy of $0.8669,0.8437,0.9015$ and 0.9960 , while SVM crossvalidation was determined with the accuracy of $0.7778,0.7206,0.9015$ and 0.9319 . (Matt et. Al, 2015). 


\subsection{Works about Deep Learning}

A study was performed to estimate the subcellular localization of the protein using deep learning. DeepPSL's SAE algorithm was implemented in MATLAB and the algorithm was accelerated in GPU. (Using NVIDIA Tesla K80). DeepPSL performance has been satisfactory. Overall performance was measured as $37.4 \%$. Compared to the method of using traditional machine learning algorithms, the results of the experiments showed that DeepPSL was better. This study also guided the comparison of deep learning and machine learning (Leyi et. Al, 2018).

A study was performed using deep learning to estimate the dose of heparizin in continuous renal replacement therapy. Experiments on test datasets showed that the deep learning architecture gave good performance and suggested that it provided at least $10 \%$ higher accuracy in the ratio of 5\% chance compared to other conventional estimation approaches (Ning et. Al, 2017).

For the early classification of renal tumor types, a different type of entrepreneurial approach, diffusion weighted MRI, was used to study the selection types. The results show that $98 \%$ of the subjects were classified as true. Therefore, the proposed approach has kept hope as a reliable non-invasive diagnostic tool (Shehata, 2016).

Renal survival was routinely examined using a Deep Neural Networks model, scoring the amount of fibrosis and tubular atrophy in the renal biopsy sample for chronic kidney injury, in a semi-quantitative manner. Although image digitization and morphometric techniques can better measure the degree of histological damage, more widely applicable network models are needed to classify the stages of kidney disease. CNN models performed better than PEFS during classification tasks. Specifically, the CNN model predicted the CKD step more accurately than the PEFS model. The area under the curve (AUC) in creatinine models was 0.912 (CNN) and 0.840 (PEFS). AUC for proteinuria models was 0.867 (CNN) versus 0.702 (PEFS), while AUC values for CNN models for 1,3 and 5 years of renal survival were $0.878,0.875$ and 0.904 , respectively, whereas AUC values for the PEFS model were $0.811,0.800$, and 0.786 . The study is presented as a proof of the principle that deep learning can be applied to routine renal biopsy images (Vijaya et. al, 2018).

Two deep learning models were adapted from a previously prepared CNN to classify glomerular segmentation and glomeruli on all slide images with frozen section. Of these models, Patch-Based Model has a mean of 0.879 , and Normalized Confusion Matrix for Patch-Based Model, with an average of 0.865 successes. This task is said to be critical in the time-sensitive assessment of donor's kidneys prior to transplantation. As a result of this study, it was agreed that it would have the potential to be an important part of the workflow for transplant evaluation in the clinical setting (Jon et. al, 2018).

In his recent paper, Kolachalamaet supported deep learning and viewed histology to classify the phenotypes of chronic kidney disease as patient-specific. This research was carried out with CNN from the most popular deep learning networks. CNNs consist of node layers, such as the conventional neural network; however, contrary to the conventional network, the CNNs assume that the input is a multichannel image. The authors wanted to investigate the utility of CNNs to classify and performed a study on the severity of chronic kidney disease from renal biopsy images. They used 171 renal biopsy training sets of varying degrees. Inception-v3, a CNN of Google, was used in this study. A pre-made architectural set has been used on millions of images from the ImageNet dataset. As a basis for comparison, CNN models outperformed the pathologist-predicted fibrosis scores for all classification tasks, and the CHD below this curve was found to be 8 to $24 \%$ higher than the equivalent of the fibrosis score estimated by the pathologist (Amelia, Averitt and Karthik , 2018).

In his study of the algebraic histogram feature model and infrequent neural network techniques for the design of kidney image classification, the kidney image was subjected to the integrated algorithm to process and detect an abnormality on the model using hardware identification language (HDL). The kidney abnormality detected in the image is indicated by color for easy identification and visualization of the abnormality on the monitor. All methods were evaluated in terms of their accuracy and yielded efficient results. The highest classification performance was achieved with $99.85 \%$ deep learning model. In addition, while calculating the similarity of kidney image classifications, it has been suggested that research can be developed by adapting various similarity criteria (Vinoth and Bommannaraja, 2017).

In a deep learning-based study to measure the level of sediment in urine, error rates during the measurement were reduced and a healthier examination result scale was aimed. In this study DenseNet to the baseline model (DFPN), deep learning model was used. The result of the study was the correct classification with 86.9\% success (Liang et. al, 2018).

In a study on the diagnosis of abnormal kidney and urinary tract disorders in children based on ultrasound imaging data, kidney images were classified. A deep learning system consisting of three different units was used. An algorithm has been developed to provide better results for kidney images (Zheng, Tasian, Fan, 2018).

In an image-based study to recognize the right and left kidneys by deep learning, a multilayered multi-layer Group Method Data Processing (GMDH) type neural network algorithm was used to identify the right and left kidney regions. The deep multilayered GMDH type neural network algorithm can automatically regulate deep neural network architectures with many hidden layers, and these deep neural networks can determine the properties of very complex nonlinear systems. In this regard, the deep learning method has once again been proven to be an accurate method for kidney images (Tadashi, Junji , 2015).

Deep learning algorithms were used to observe capillary and inflammation formation in frozen kidneys for transplantation. In this study, the development of a deep learning model that describes and classifies sclerosis and non-sclerosis glomeruli in all slide images of the donor kidney's frozen section biopsies is explained. This model is extended to a pre-trained convolutional neural network $(\mathrm{CNN})$ in a large database of digital images. The deep learning models used in the study showed that $97 \%$ of kidney images were and these results were very important for kidney transplantation (Jon et. al, 2018). 


\section{Results}

Cancer is an important health problem worldwide. Although methods of artificial intelligence learning are more widely used in cancer prediction, none of the methods make the others completely ineffective.

Due to the complexity of cancer and the high mortality rate, timely and accurate diagnosis is important. Specifically, kidney cancer types may not show symptoms from the last stages and even cancer without spreading to the other organs of the body. So it is extremely important in the treatment of cancer to improve the accuracy of estimation by applying improved and up-to-date techniques. Numerous studies have been carried out on various types of cancer in recent years, especially with machine learning and deep learning methods. As we have seen from these studies, each new study not only supports the previous one, but also aimed to find its deficiencies. In our literature study, the lack of studies on kidney tumors has encouraged us to address this issue. There is a lack of data in the studies. Diagnosis and classification of kidney tumors will provide more successful results with machinery and deep learning methods, especially when working with more images. What we want to pay attention here is to achieve success in treatment by developing an appropriate algorithm for early diagnosis using machine learning and deep learning methods. In recent years, various machine-learning techniques have been used for the diagnosis and prediction of nephrology. Each problem requires an understanding of the problem to diagnose and diagnose the most appropriate machine learning algorithm.

Machine learning and deep learning are usually nonlinear analysis and have many parameters and multiple layers. Especially the big data and the unchecked features (such as high blood pressure affecting the kidney) do not give any successful results with other methods, but they give quite successful results with machine learning and deep learning methods. Using machine learning and deep learning methods, complex relationships between classes are automatically learned. Thus machine learning and deep learning methods achieves a better result. In the near future, computers with machine learning and deep learning systems in health care facilities will be a standard for decision making and disease diagnosis. Of course, it should be noted that expert opinions should be taken into consideration here.

Although machine learning and deep learning methods are now the best performance of most medical image analysis and comparison of medical diagnostic methods, it is not correct to say that this is the exact result. Therefore, the design of the system will be combined with all other external factors to make a definite conclusion. In this respect, the experience of the experts on the subject, the physician information and more data sets are important factors that will increase the success of the study.

\section{References}

Ahmed J., Aljaaf and friends. (2018). Early Prediction of Chronic Kidney Disease Using Machine Learning Supported by Predictive Analytics. IEEE Evrimsel Hesaplama Kongresi (CEC). pp. 1-9.

Amelia, J., Averitt and Karthik, N. (2018). Going Deep: The Role of Neural Networks for Renal Survival and Beyond, Kidney IntRep, 3, pp.242-243.

American Cancer Society. (2008). Estimated New Cancer Cases and Deaths by Gender in All Regions. Retrieved from www.cancer.org.

Anusorn, C., Thipwan F., and friends. (2016). Predictive Analytics for Chronic Kidney Disease Using Machine Learning Techniques, MITICON.

Bengio, Y. P., Lamblin, Popovici, D. and Larochelle, H. (2006). Greedy layer-wise training of deep networks. Proceedings of the 19th International Conference on Neural Information Processing Systems. MIT Press, pp. 153-160.

Carreira, M. A. and Hinton, G. E. (2005). On Contrastive Divergence Learning. Artif. Intell. Stat., Vol. 10.

Chi-Jim, C., Tun-Wen, P. and friends. (2014). Stage Diagnosis for Chronic Kidney Disease Based on Ultrasonography. 11th International Conference on Fuzzy Systems and Knowledge Discovery.

Doğruyol, M., Aydın, A. (2017). Topluluk sınıflandırıcılarını kullanarak kronik böbrek hastalığının saptanması. ELECO 2017, pp. 544-547.

Elman, J. L. (1991). Finding Structure in Time. Cogn. Sci., vol. 14, No.2, pp. 179-211.

Emily, S. Blum and friends. (2018). Early Detection of Ureteropelvic Junction Obstruction Using Signal Analysis and Machine Learning. The journal of Urology, pp.847-852.

Eroğlu, K., Palabaş, T. (2016). Kronik Böbrek Hastalığı Tespitinde Farklı Sınıflandırma Yöntemleri ve Farklı Topluluk Algoritmalarının Birlikte Kullanımının Sinıflandırma Performansına Etkisi., ELECO, pp. 512 - 516.

Eskisehir. (2017). Anadolu International Conference in Economics V, May 11-13, Eskisehir, Turkey.

Fukushim, K. N. (1980). A self-organizing neural network model for a mechanism of pattern recognition unaffected by shift in position. Biol. Cybern., Vol. 36, No. 4, pp. 193-202.

Hinton, G. E. and Salakhutdinov, R. R. (2006). Reducing the Dimensionality of Data with Neural Networks. Science (80-.), Vol. 313, No.5786, pp. 504-507. 
Hinton, G. E., Osindero, S. and the, Y.W. (2006). A Fast Learning Algorithm for Deep Belief Nets. Neural Comput. Vol. 18, No. 7, pp. 1527-1554.

Hubel, D. H. and Wiesel, T. N. (1968). Receptive fields and functional architecture of monkey striate cortex. J. Physiol., Vol. 195, pp. 215-243.

Jon N. M. and friends. (2018). Deep Learning Global Glomerulosclerosis in Transplant Kidney Frozen Sections, IEEE Transactions On Medical Imaging.

Jon, N. M., Matthew, K., Satoru, K., Ta-Chiang, L., Thaddeus, S., Stappenbeck, Joseph P. G., and Joshua, S., Swamidass. (2018). Deep Learning Global Glomerulosclerosis in Transplant Kidney Frozen Sections.

Koçak, B. and friends. (2018). Renal hucrelik karsinom alt tipleri arasındaki dokusal farklılıklar, Avrupa Radyoloji Dergisi, pp. 149-157.

Krizhevsky, A. and Hinton, G. E. (2011). Using Very Deep Autoencoders for Content Based Image Retrieval. In European Symposium on Artificial Neural Networks, pp. 489-494.

Lecun, Y., Bottou L., Bengio Y., and Haffner, P. (1998). Gradient-based learning applied to document recognition. Proc. IEEE, Vol. 86, No. 11, pp. 2278-2324.

Leyi, W., Yijie, D., Ran, S., Jijun, T., Quan, Z. (2018). Parallel Distrib. Comput.212-217.

Liang, Y., Tang, Z., Meng, Y., Liu, J. (2018). Object detection based on deep learning for urinesediment examination, biocybernetics and biomedical engineering. pp.661-670.

Lorenzo, A. J., Rickard, M., Luis, H., Braga, Y., Oliveria,, J. (2018). Predictive Analytics and Modeling Employing Machine Learning Technology, Urology.

Matt., S., Martin P., John, H., Phanl, and May D. (2015). Integration of Multimodal RNA-Seq Data for Prediction of Kidney Cancer Survival. IEEE International Conference on Bioinformatics and Biomedicine (BTBM).

Mehmet Akif Ersoy U. (2017). Artificial Intelligence and Machine Learning Applications in Big Data Analysis. Journal of Social Sciences Institute. Vol. 9, No.22, pp.155-172.

Mercaldo, F., Nardone, V., Santone, A. (2017). Diabetes Mellitus Affected Patients Classification and Diagnosis through Machine Learning Techniques, International Conference on Knowledge Based and Intelligent Information and Engineering Systems, KES2017, Marseille, France.

Mikolov, T. (2010). Recurrent neural network based language model, in Interspeech,

Monika, R. and Uvais, Q. (2003). Yıllık Teknik Konferans IEEE Bölgesi 5, pp. 23-27.

Muratbinbay. (2018). Retrieved from https://www.muratbinbay.com.

Ning, K., Xiaoxi, L., Chunyan, L., Jie, L., Hongwei, W. (2017). Deep architecture for Heparin dosage prediction during continuous renal replacement therapy. Proceedings of the 36th Chinese Control Conference July 26-28. Dalian, China.

Onkoloji. (2018). Retrieved from http://www.onkoloji.gov.tr.

Qiang, Z., Gregory, T., Yong F. (2018). Transfer Learning For Diagnosis Of Congenital Abnormalities Of The Kidney And Urinary Tract In Children Based On Ultrasound Imaging Data, ISBI.

Ranzato, M., Huang, F. J., Boureau, Y.L. and LeCun, Y. (2007). Unsupervised Learning of Invariant Feature Hierarchies with Applications to Object Recognition. In 2007 IEEE Conference on Computer Vision and Pattern Recognition, pp. 1-8.

Roberto, A., Remi, C., Ketan, B., Vincent, A. (2015). Fast Kidney Detection and Segmentation with Learned Kernel Convolution and Model Deformation in 3d Ultrasound Images. Medisys Research Lab, Philips Research, Suresnes, France.

Salakhutdinov, R. and Hinton, G. (2009). Deep Boltzmann Machines,"in International Conference on Artificial Intelligence and Statistics", pp. 3- 11.

Seker, A. and Gürkan, A. (2017). Yuksek, Stacked Autoencoder Method for Fabric Defect Detection. Sci. Sci. J., Vol. 38 , No. 2.

Seker, A. Diri, B., Balik, H. (2017). Derin Öğrenme Yöntemleri ve Uygulamaları Hakkında Bir İnceleme. Gazi Mühendislik Bilimleri Dergisi, 3, pp.47-64.

Shehata, M. And friends. (2016). A new non-invasive approach for early classification of renal rejection types using diffusionweighted mri, icip.

Smolensky, P. (1986). Information Processing in Dynamical Systems: Foundations of Harmony Theory.

Tadashi, K., Junji, U. (2015). The 3-Dimensional Medical Image Recognition of Right and Left Kidneys by Deep GMDH-type Neural Network. Track3: Bioinformatics, Medical Imaging and Neuroscience, Okinawa, Japan, ICIIBMS.

Tibeter. (2018). Retrieved from https://tibeterdogru.com.

Uroweb. (2018). Retrieved from https://patients.uroweb.org/en/ben-bir-uroloji-hastasiyim/bobrekkanseri/bobrek-kanseri-tani-vesiniflamasi/siniflama. 
Vijaya, B., and friends. (2018). Renal Survival Using Deep Neural Networks, Kidney IntRep 3, pp.464-475.

Vinoth, R., and Bommannaraja, K. (2017). FPGA Design of Efficient Kidney Image Classification using Algebric Histogram Feature Model and Sparse Deep Neural Network (SDNN) Techniques. Conference on Emerging Devices and Smart Systems (ICEDSS).

Zheng, Q., Tasian, G., Fan, Y. (2018). Transfer learnıng for diagnosis of congenital abnormalities of the kidney and urınary tract in children based on ultrasound imaging data. International Symposium on Biomedical Imaging. 